Article

\title{
Exploiting the Advantages of Molecular Tools for the Monitoring of Fungal Indoor Air Contamination: First Detection of Exophiala jeanselmei in Indoor Air of Air-Conditioned Offices
}

\author{
Xavier Libert ${ }^{1,2}{ }^{\text {, Camille Chasseur }}{ }^{3}$, Ann Packeu ${ }^{3}$, Fabrice Bureau ${ }^{2}$, Nancy H. Roosens ${ }^{1}$ and \\ Sigrid C. J. De Keersmaecker $1, *$ (D) \\ 1 Transversal activities in Applied Genomics, Sciensano, J. Wytsmanstraat 14, 1050 Brussels, Belgium; \\ libert.xavier85@gmail.com (X.L.); nancy.roosens@sciensano.be (N.H.R.) \\ 2 Cellular and Molecular Immunology, Groupe Interdisciplinaire de Génoprotéomique Appliquée (GIGA), \\ Université de Liège (ULg), Avenue de l'Hôpital, 1 (B34), 4000 Sart-Tilman, Belgium; fabrice.bureau@ulg.ac.be \\ 3 Mycology and Aerobiology, Sciensano, J. Wytsmanstraat 14, 1050 Brussels, Belgium; \\ Camille.Chasseur@sciensano.be (C.C.); ann.packeu@sciensano.be (A.P.) \\ * Correspondence: Sigrid.dekeersmaecker@sciensano.be; Tel.: +32-2-642-5257
}

Received: 7 November 2019; Accepted: 9 December 2019; Published: 10 December 2019

check for updates

\begin{abstract}
Today, indoor air pollution is considered a public health issue. Among the impacting pollutants, indoor airborne fungi are increasingly highlighted. Most of the monitoring protocols are culture-based, but these are unable to detect the uncultivable and/or dead fraction or species suppressed by fast-growing fungi, even though this fraction could impact health. Among the contaminants suspected to be part of this fraction, Exophiala jeanselmei is an interesting case study. Known to be pathogenic, this black yeast grows in humid environments such as air-conditioning systems, where it has been previously detected using classical culture-based methods. However, until now, this fungus was never detected in indoor air in contact with these air-conditioning systems. This study shows the first detection of E. jeanselmei in indoor air collected from offices in contact with contaminated air-conditioning reservoirs. While its presence in indoor air could not be demonstrated with culture-based methods, it was found by real-time PCR and massive parallel sequencing. The latter also allowed obtaining a broader view on the fungal diversity in the tested samples. Similar approaches were applied on water samples collected from the conditioning reservoirs to trace the source of contamination. The comparison of results obtained with both methods confirmed that the molecular tools could improve indoor air monitoring, especially of dead and/or uncultivable contaminants or when competition between species could occur.
\end{abstract}

Keywords: Exophiala jeanselmei; indoor air contamination; real-time PCR; NGS; detection; public health; identification; molecular methods

\section{Introduction}

Today, as most of their time is spent inside homes, offices, and other workplaces, people are increasingly impacted by a low indoor air quality. Among the different indoor pollutants inventoried, fungi and molds appear to be important airborne contaminants in workplaces, houses, and buildings, and they are suspected to have an impact on health, especially on respiratory diseases such as allergy, asthma, asthma exacerbation, or rhinitis. In this way, indoor fungal contamination is more and more considered as a public health issue by the scientific community [1-6] and by preventive health care actors such as environmental agencies $[7,8]$ and the World Health Organization [9]. These fungal contaminants 
can originate from different sources, i.e., they can enter from outside environments, via windows, aeration, and ventilation, such as is the case for Alternaria alternata and Cladosporium [10-14] species, or they can occur in water contained in reservoirs in air-conditioning systems. One such example is Exophiala jeanselmei, a black yeast known as a pathogenic species associated, amongst others, with cutaneous infections, subcutaneous cysts, and systemic and nosocomial infections [15-18]. This species, living in humid and oligotrophic environments, is observed in feed, sludge, and stagnant water [19]. Recently, E. jeanselmei has been detected for the first time in outdoor and at low levels in indoor air sampled in 2012-2015 at different locations in the areas of arctic settlement Tiksi (Russian Arctic) [20]. In indoor environments, E. jeanselmei is also detected in sludge piping, water systems (pipes, bathroom, reservoir, sauna, etc.) and water reservoirs of air-conditioning systems [17,19,21,22]. For some other species, it has been postulated that the mechanism of indoor air contamination is linked to these water reservoirs of air-conditioning units or pipings [12]. However, although E. jeanselmei can be detected in humid indoor environments (i.e., water samples taken from water reservoirs of air-conditioning systems), it has never been found before in indoor air in contact with these air-conditioning systems during the air-conditioning water monitoring campaign. It can be hypothesized that this is due to the disadvantages of the currently used monitoring methods. Indeed, routine protocols for indoor air fungal monitoring are still based on culture, colony counting, and microscopic visualization. Therefore, the results of these methods are dependent on the culture conditions, such as the selection of the medium, species competition (especially a problem of suppression by fast-growing fungi during long-term incubation periods), growth conditions, and differences in terms of incubation time $[23,24]$. Moreover, culture-based protocols are not able to detect the uncultivable and/or dead fraction (i.e., fragments of mycelia or cell walls, dead cells, etc.) which could be airborne and collected during the sampling $[23,24]$. However, this fraction of suppressed or uncultivable species could have an impact on health and should, therefore, also be monitored; otherwise, important elements are missing to establish the scientific link between fungal airborne contamination and health problems. This could especially be true for E. jeanselmei. The monitoring of this fungus in water samples is complicated, as it needs up to 21 days to grow in culture [25], while being highly demanding in terms of culture conditions, i.e., growth medium chosen, competition between species, temperature, and humidity. Especially, this last factor can impede the detection of E. jeanselmei in indoor air, as the fact that it might occur in a desiccated form will hamper its cultivation, and hence its detection, using the classical monitoring procedures.

The use of molecular methods, such as polymerase chain reaction (PCR), real-time PCR (RT-PCR), and sequencing, could offer a solution to investigate the indoor airborne fungal community, including this suppressed, uncultivable, and/or dead fraction [23,24,26-30]. We have previously developed a qRT-PCR-based assay for the detection of E. jeanselmei in water samples [31]. In the present study, we have tried this method on air samples collected with the Coriolis ${ }^{\circledR} \mu$ air sampler from different offices in contact with air-conditioning reservoirs contaminated with E. jeanselmei. The aim was to investigate the hypothesis that this species is occurring in indoor air as an out-competed or uncultivable and/or dead fraction, i.e., that it is not detectable with the classical monitoring methods based on culturing, but that it can be detected using molecular, i.e., DNA-based, technologies. To demonstrate that massive parallel sequencing could improve our insight into the fraction of uncultivable fungi, the RT-PCR analysis was complemented with massive parallel sequencing of the DNA extracted from the air and water samples.

\section{Materials and Methods}

\subsection{Sampling}

All the samples (i.e., air and water samples) were collected between February 12th and March 13th, 2015 in Brussels, Belgium. 
The reservoir of water from five air-conditioning systems, as seen in Table 1, for which previous monitoring results had pointed to E. jeanselmei contamination, was investigated using the sampling protocol of water from air-conditioning reservoirs previously described [32]. In short, $1 \mathrm{~L}$ of water situated between $1 \mathrm{~cm}$ and $5 \mathrm{~cm}$ above the bottom of the water reservoir situated in the pulse group (PG, i.e., the part of the air-conditioning system containing the machinery to pulse the air and the water-reservoir needed to maintain the required hygrometry level) was put into a sterile Duran bottle. All samples were kept at $4{ }^{\circ} \mathrm{C}$ until their analysis.

Table 1. Detection of E. jeanselmei in indoor air and water in air-conditioning systems: Classical analysis and RT-PCR.

\begin{tabular}{|c|c|c|c|c|c|c|}
\hline \multirow{3}{*}{ Sample ID * } & \multirow{3}{*}{ Pulse Group } & \multirow{3}{*}{ Office } & \multirow{3}{*}{ Sample Type } & \multicolumn{3}{|c|}{ Analysis Method } \\
\hline & & & & \multirow{2}{*}{$\begin{array}{c}\text { Culture } \\
\text { CFU/mL } \\
1\end{array}$} & \multicolumn{2}{|c|}{ RT-PCR } \\
\hline & & & & & $\operatorname{Tm}\left({ }^{\circ} \mathrm{C}\right)^{2}$ & $\mathrm{Cq}^{3}$ \\
\hline PG1 4 & 1 & & Water ${ }^{5}$ & 0 & N/A & N/A \\
\hline PG1-1 & 1 & 1 & Air & 0 & N/A & N/A \\
\hline PG1-2 & 1 & 2 & Air & 0 & N/A & $\mathrm{N} / \mathrm{A}$ \\
\hline PG1-3 & 1 & 3 & Air & 0 & N/A & N/A \\
\hline PG2 & 2 & & Water ${ }^{5}$ & 10 & $79.53 \pm 0.12$ & $28.00 \pm 1.35$ \\
\hline PG2-1 & 2 & 1 & Air & 0 & N/A & N/A \\
\hline PG2-2 & 2 & 2 & Air & 0 & N/A & N/A \\
\hline PG2-3 & 2 & 3 & Air & 0 & N/A & N/A \\
\hline PG3 & 3 & & Water ${ }^{5}$ & 10 & $79.79 \pm 0.32$ & $28.39 \pm 0.93$ \\
\hline PG3-1 & 3 & 1 & Air & 0 & $\mathrm{~N} / \mathrm{A}$ & $\mathrm{N} / \mathrm{A}$ \\
\hline PG3-2 & 3 & 2 & Air & 0 & N/A & N/A \\
\hline PG3-3 & 3 & 3 & Air & 0 & N/A & N/A \\
\hline PG4 & 4 & & Water ${ }^{5}$ & 100 & $79.93 \pm 0.05$ & $20.32 \pm 1.98$ \\
\hline PG4-1 & 4 & 1 & Air & 0 & $79.44 \pm 0.10$ & $23.79 \pm 0.92$ \\
\hline PG4-2 & 4 & 2 & Air & 0 & $79.49 \pm 0.03$ & $24.49 \pm 0.23$ \\
\hline PG4-3 & 4 & 3 & Air & 0 & $79.38 \pm 0.03$ & $34.05 \pm 1.75$ \\
\hline PG5 & 4 & & Water ${ }^{5}$ & 15 & $79.34 \pm 0.23$ & $27.36 \pm 1.12$ \\
\hline PG5-1 & 5 & 1 & Air & 0 & $79.39 \pm 0.02$ & $27.62 \pm 0.63$ \\
\hline PG5-2 & 5 & 2 & Air & 0 & N/A & N/A \\
\hline PG5-3 & 5 & 3 & Air & 0 & N/A & N/A \\
\hline
\end{tabular}

* $\mathrm{PG}=$ pulse group, i.e., the part of the air-conditioning system containing the machinery to pulse the air and the water-reservoir needed to maintain the required hygrometry level; the first number refers to the number of the PG (five in total); the number after the '-' refers to the office connected to the PG, at different distances (see Materials and Methods). ${ }^{1} \mathrm{CFU} / \mathrm{mL}$ defined as the number of colonies of $E$. jeanselmei on plate after 21 days of incubation at $37^{\circ} \mathrm{C} .{ }^{2} \mathrm{Tm}$ defined as the average of the melting temperature $\left({ }^{\circ} \mathrm{C}\right)$ observed for each RT-PCR amplicon obtained during the qPCR SYBR ${ }^{\circledR}$ Green analysis performed in duplicate in two independent runs. ${ }^{3} \mathrm{Cq}$ defined as the average quantification cycle observed for each RT-PCR amplicon obtained during the qPCR SYBR ${ }^{\circledR}$ Green analysis performed in duplicate in two independent runs. ${ }^{4}$ PG1 was maintained before the sampling. ${ }^{5}$ One sample per pulse group was analyzed.

In addition to the water sampling, air sampling was done in the offices connected to the five air-conditioning systems of which water samples were collected (PG1 to PG5). For each of the air-conditioning systems, three offices were selected based on their distance to the PG, i.e., the closest $(-1)$ and most distantly located (-3) office relative to the PG and one office in between the two other offices (-2). Each sample was taken in duplicate. The indoor air samples were collected with a Coriolis ${ }^{\circledR}$ $\mu$ air sampler (Bertin Technology, Motigny-le-Bretonneux, France) following the sampling protocol reported by Libert and colleagues in 2015 [33].

\subsection{Classical Analysis}

The protocol used for the classical analysis (i.e., culture and fungal determination) of the water and air samples, collected with the Coriolis ${ }^{\circledR} \mu$ air sampler, has been previously described [31-33]. According to the protocols, the incubation time ranged between five and 21 days as recommended for the detection of major indoor air species and for the detection of E. jeanselmei. After five days of 
incubation, the plate was analyzed as it was recommended for the detection of indoor air species. After this microscopic analysis, the plate was reincubated for 16 days (total incubation time 21 days) as is recommended for the detection of E. jeanselmei.

\subsection{DNA Extraction}

The protocol used for the DNA extraction from water [31] and indoor air samples [33] was previously described. This extraction protocols have been optimized in order to avoid inhibition in downstream PCR-based analysis. Genomic DNA amount and purity were evaluated with a Nanodrop ${ }^{\circledR}$ 2000 (Thermo Scientific, Wilmington, DE, USA).

\subsection{RT-PCR Screening}

The analyses of DNA extracted from air and water samples were performed in duplicate in two independent runs. The RT-PCR assay used was the SYBR ${ }^{\circledR}$ Green Ejeanselmei_ITS assay earlier reported for the detection of E. jeanselmei in water [31]. This assay uses the SYBR ${ }^{\circledR}$ green PCR Mastermix (Diagenode, Liège, Belgium). The sequences of the primers used (targeting the internal transcribed spacer region (ITS)) are provided by Libert and colleagues [31]. The primers were purchased from Eurogentec (Liège, Belgium). Each run was done on a CFX TouchTM Real-Time PCR detection System equipped with CFX Manager software V. 2 (Biorad, Temse, Belgium) and was carried out with $5 \mu \mathrm{L}$ of a positive control (PC), corresponding to 200 theoretical copies of gDNA of E. jeanselmei strain BCCM/IHEM 4740 purchased from the BCCM/IHEM collection of Sciensano in Brussels (Belgium). A "no template control" (NTC), i.e., using Gibco ${ }^{\circledR}$ DNase, RNase, Protease free pure water (Life Technologies, Gent, Belgium) as template, was also added in order to verify that no contamination occurred.

In order to confirm the identity of each RT-PCR amplicon, Sanger sequencing analysis was performed on an ABI3130xl Genetic Analyzer apparatus (Applied Biosystem, Life Technologies, Gent, Belgium) with the BigDye Terminator v3.1 cycle sequencing kit (Applied Biosystems) according to the manufacturer's instructions. Each sequence was identified by comparison to the sequences available in the NCBI database using the BLASTn tool (http://blast.ncbi.nlm.nih.gov/).

\subsection{Massive Parallel Sequencing}

To confirm the identity of RT-PCR amplicons, but also to demonstrate that massive parallel sequencing could be useful for the investigation of uncultivable and dead species present in the air and water samples, all the samples were sequenced using an Illumina MiSeq instrument.

Because a massive parallel sequencing analysis using the Illumina MiSeq with a MiSeq reagent kit v3 reaches its optimal performance with fragments no longer than $600 \mathrm{bp}$ and because the fungal ITS region has a length around $800 \mathrm{bp}$, the PCR amplicons for library preparation were prepared for both the ITS- 1 and ITS-2 region using DNA extracted from different environmental (water and air) samples. Hereto, amplification with the universal forward (f) and reverse (r) primers ITS1f/ITS2r39 for the amplification of the ITS-1 region and with the universal primers ITS3f/ITS4r39 for the ITS-2 region was performed [34]. The primers used were extended with the Illumina sequences needed for PCR-based library preparation. The PCR mix ( $25 \mu \mathrm{L}$ final volume) contained $2.5 \mu \mathrm{L}$ of high fidelity PCR buffer (10X), $0.5 \mu \mathrm{L}$ of a mix of $0.2 \mathrm{mM}$ of each dNTP (ThermoFisher Scientific, Gent, Belgium), $0.5 \mu \mathrm{M}$ of each primer, $0.1 \mu \mathrm{L}$ of Platinum ${ }^{\circledR}$ Taq DNA Polymerase high fidelity enzyme $(5 \mathrm{U} / \mu \mathrm{L})$ (ThermoFisher Scientific, Life Technology, Gent, Belgium), and $15.90 \mu \mathrm{L}$ of Gibco ${ }^{\circledR}$ DNase, RNase, Protease free pure water (Life Technologies, Gent, Belgium). At the end, $5 \mu \mathrm{L}$ of gDNA (10 ng) were added. Each run was performed with following PCR protocol, i.e., one cycle at $94^{\circ} \mathrm{C}$ for $3 \mathrm{~min}, 35 \mathrm{cycles}$ of $30 \mathrm{sec}$ at $94{ }^{\circ} \mathrm{C}$, $30 \mathrm{sec}$ at $55^{\circ} \mathrm{C}$ and $1 \mathrm{~min}$ at $72{ }^{\circ} \mathrm{C}$, and at last, $10 \mathrm{~min}$ at $72{ }^{\circ} \mathrm{C}$.

After the PCR, all samples were purified with the AMpure ${ }^{\circledR}$ XP PCR purification kit (Agencourt Bioscience corporation, Beverly, MA, USA). The quality and amount of PCR fragments for massive parallel sequencing was verified on a Bioanalyzer 2100 (Agilent Technologies, Amstelveen, the Netherlands). ITS-1 and ITS-2 amplicons from a same sample were mixed together. Equimolar mixes were made 
according to the size of the peaks observed during the Bioanalyzer analysis. Sequencing was performed by BaseClear (Venlo, The Netherlands) with an Illumina MiSeq, yielding $2 \times 300$ bp paired-end reads.

\subsection{Bioinformatics Analysis}

The FASTQ sequence files were generated using the Illumina Casava pipeline version 1.8.3 (BaseClear, Venlo, the Netherlands). The first quality assessment was based on the Illumina Chastity filtering. Reads with PhiX control signal and/or short reads below $20 \mathrm{bp}$ (after adapter clipping) were removed using an in-house filtering protocol from BaseClear. Then, a second quality assessment control was done using the tool PRINSEQ-lite quality control v.0.20.4. Finally, an average quality score per sample was obtained with FastQC v.010.1.40 [35]. The sequences which did not pass this quality control were removed for the remainder of the analysis.

To determine the presence of E. jeanselmei and other fungal species in indoor air and water samples, sequencing reads were analyzed with an operational taxonomic unit (OTU) classification approach with the Microbial Genomics Module from the CLC Genomic Workbench V8 software (Qiagen Benelux, B.V., KJ Venlo, the Netherlands). Briefly, in order to have comparable read lengths and to remove reads with low coverage, the paired-end reads were firstly treated with three different tools, i.e., "adapter trimming", "fixed length trimming", and "filter samples based on the number of reads". Thus, at the end of the workflow, the OTU clustering was performed only on reads with the same length and a low level of bias.

The OTUs clustering was made with the OTUs clustering tool. The database used as reference was the fungal rDNA sequences Database UNITE V7.1. [36]. Finally, all the OTUs were aligned with the MUSCLE tool and a Neighbor Joining tree was constructed according to the Jukes Cantor nucleotides model.

\section{Results}

\subsection{Classical Analysis by Culturing}

The sampling of the offices and water reservoirs of the air-conditioning systems was performed as elaborated in Materials and Methods. The water and indoor air samples were both incubated on plate in order to detect $E$. jeanselmei, as outlined in the classical monitoring procedures.

For the five water samples collected from the pulse group (PG) of the air-conditioning systems, E. jeanselmei was detected in four of them, as seen in Table 1. Expressed in colony forming units per $\mathrm{mL}$ $(\mathrm{CFU} / \mathrm{mL})$, the range of contamination of the water samples was determined to be between 10 and $100 \mathrm{CFU} / \mathrm{mL}$, as seen in Table 1. E. jeanselmei colonies were not observed for the sample collected from the PG 1, as seen in Table 1. In addition, three other determined taxa and three nonsporulating sp. or undetermined species were detected on plates, i.e., Aspergillus fumigatus, Aspergillus puulaeunsis, and Penicillium chrysogenum, as seen in Table 2. As it was done for the water samples, the indoor air samples collected in each office were incubated according to the protocol developed for the detection of E. jeanselmei [36,37]. As shown in Table 1, after 21 days of incubation, no E. jeanselmei was detected on plate for any of the offices in contact with the air-conditioning system. However, four other determined taxa and four nonsporulating sp. or undetermined species were observed on plate, i.e., A. alternata, Aspergillus sp., A. versicolor, and P. chrysogenum, as seen in Table 3. 
Table 2. Fungal contamination in water samples, other than E. jeanselmei: Classical analysis and sequencing analysis comparison.

\begin{tabular}{|c|c|c|c|c|}
\hline \multirow[b]{2}{*}{ Sample * } & \multicolumn{2}{|l|}{ Classical Analysis ${ }^{1}$} & \multicolumn{2}{|l|}{ NGS } \\
\hline & Species & $\mathrm{CFU} / \mathrm{mL}$ & Species & $\begin{array}{l}\text { Abundance of Reads } \\
\text { Per OTU }{ }^{2}(\%)\end{array}$ \\
\hline PG1 & Penicillium chrysogenum & 5 & Penicillium chrysogenum & 100 \\
\hline \multirow{4}{*}{ PG2 } & Penicillium chrysogenum & 5 & Penicillium chrysogenum & 60.64 \\
\hline & Aspergillus puulaaeunsis & 1 & Aspergilluspuulaaeunsis & 11.27 \\
\hline & Nonsporulating sp. & 1 & & \\
\hline & Aspergillus fumigatus & 4 & Aspergillus fumigatus & 36.34 \\
\hline \multirow[t]{3}{*}{ PG3 } & Penicilliumchrysogenum & 5 & Penicilliumchrysogenum & 30.15 \\
\hline & Nonsporulating sp. & 2 & & \\
\hline & Undetermined species & 1 & & \\
\hline PG4 & Aspergillus fumigatus & 2 & Aspergillus fumigatus & 12.19 \\
\hline \multirow[t]{2}{*}{ PG5 } & Penicillium chrysogenum & 3 & Penicillium chrysogenum & 23.30 \\
\hline & & & Aspergillus puulaaeunsis & 11.86 \\
\hline
\end{tabular}

* PG = pulse group, i.e., the part of the air-conditioning system containing the machinery to pulse the air and the water-reservoir needed to maintain the required hygrometry level; the first number refers to the number of the PG (5 in total). ${ }^{1} \mathrm{CFU} / \mathrm{mL}$ defined as the number of colonies observed on plate after five days and 21 days of incubation at $37^{\circ} \mathrm{C}$, E. jeanselmei excluded. The data for E. jeanselmei is presented in Table $1 .{ }^{2}$ The massive parallel sequencing data were analyzed with the CLC Genomic Workbench software (Qiagen Benelux, B.V., KJ Venlo, the Netherlands) and the Microbial Genomics Module. The database used as reference was the UNITE database ${ }^{36 .}$

Table 3. Fungal contamination in air samples, other than E. jeanselmei: Classical analysis and sequencing analysis comparison.

\begin{tabular}{|c|c|c|c|c|}
\hline \multirow[b]{2}{*}{ Samples * } & \multicolumn{2}{|l|}{ Classical Analysis $^{1}$} & \multicolumn{2}{|l|}{$\mathrm{NGS}^{2}$} \\
\hline & Species & $\mathrm{CFU} / \mathrm{mL}$ & Species & $\begin{array}{l}\text { Abundance of Reads } \\
\text { Per OTU }^{2}(\%)\end{array}$ \\
\hline \multirow[t]{3}{*}{ PG1-1 } & Alternaria alternata & 5 & Alternaria alternata & 21.86 \\
\hline & Aspergillusversicolor & 3 & Aspergillus subversicolor & 11.49 \\
\hline & Penicillium chrysogenum & 9 & Penicillium chrysogenum & 66.65 \\
\hline \multirow[t]{4}{*}{ PG1-2 } & Alternaria alternata & 3 & Alternaria alternata & 24.72 \\
\hline & Aspergillus sp. & 2 & Aspergillus monodii & 7.70 \\
\hline & Nonsporulating sp. & 1 & Aspergillus rugulosus & 4.43 \\
\hline & Penicillium chrysogenum & 7 & Penicillium chrysogenum & 62.40 \\
\hline \multirow[t]{4}{*}{ PG1-3 } & Alternaria alternata & 2 & Alternaria alternata & 31.28 \\
\hline & Undetermined species & 1 & Aspergillus monodii & 15.93 \\
\hline & Nonsporulating sp. & 1 & Aspergillus rugulosus & 12.47 \\
\hline & Penicillium chrysogenum & 7 & Penicillium chrysogenum & 39.58 \\
\hline \multirow[t]{3}{*}{ PG2-1 } & Undetermined sp. & 2 & Emericella olivicola & 16.22 \\
\hline & Penicillium chrysogenum & 6 & Penicillium chrysogenum & 71.44 \\
\hline & & & Emericella undulata & 12.35 \\
\hline \multirow[t]{2}{*}{ PG2-2 } & Alternaria alternata & 2 & Alternaria alternata & 24.59 \\
\hline & Penicillium chrysogenum & 6 & Penicillium chrysogenum & 75.41 \\
\hline \multirow[t]{4}{*}{ PG2-3 } & Alternaria alternata & 4 & Alternaria alternata & 32.28 \\
\hline & Aspergillus versicolor & 5 & Aspergillus subversicolor & 32.09 \\
\hline & Aspergillus sp. & 1 & Aspergillus monodii & 24.26 \\
\hline & Nonsporulating sp. & 1 & Aspergillus puulaaeunsis & 11.37 \\
\hline \multirow[t]{2}{*}{ PG3-1 } & Alternaria alternata & 4 & Alternaria alternata & 53.50 \\
\hline & Penicillium chrysogenum & 5 & Penicillium chrysogenum & 46.50 \\
\hline \multirow[t]{3}{*}{ PG3-2 } & Alternaria alternata & 5 & Alternaria alternata & 71.96 \\
\hline & Undetermined sp. & 2 & Emericella olivicola & 16.41 \\
\hline & & & Emericella undulata & 11.63 \\
\hline \multirow[t]{4}{*}{ PG3-3 } & Aspergillus sp. & 2 & Aspergillus monodii & 11.18 \\
\hline & & & Aspergillus rugulosus & 19.56 \\
\hline & & & Aspergillus subversicolor & 20.40 \\
\hline & Penicillium chrysogenum & 6 & Penicillium chrysogenum & 48.85 \\
\hline \multirow[t]{3}{*}{ PG4-1 } & Alternaria alternata & 1 & Alternaria alternata & 26.18 \\
\hline & Aspergillus versicolor & 1 & Undertermined sp. & 13.07 \\
\hline & Nonsporulating sp. & 1 & & \\
\hline
\end{tabular}


Table 3. Cont.

\begin{tabular}{|c|c|c|c|c|}
\hline \multirow[b]{2}{*}{ Samples * } & \multicolumn{2}{|l|}{ Classical Analysis $^{1}$} & \multicolumn{2}{|l|}{ NGS $^{2}$} \\
\hline & Species & $\mathrm{CFU} / \mathrm{mL}$ & Species & $\begin{array}{l}\text { Abundance of Reads } \\
\text { Per OTU }{ }^{2}(\%)\end{array}$ \\
\hline \multirow[t]{2}{*}{ PG4-2 } & Alternaria alternata & 3 & Alternaria alternata & 17.48 \\
\hline & $\begin{array}{l}\text { Penicillium chrysogenum } \\
\text { Undetermined sp. }\end{array}$ & 9 & Penicillium chrysogenum & 40.88 \\
\hline \multirow[t]{2}{*}{ PG4-3 } & Aspergillus versicolor & 4 & Aspergillus subversicolor & 29.12 \\
\hline & Nonsporulating sp. & 1 & $\begin{array}{c}\text { Aspergillus puulaaeunsis } \\
\text { Aspergillus monodii }\end{array}$ & $\begin{array}{c}24.38 \\
7.17\end{array}$ \\
\hline \multirow[t]{3}{*}{ PG5-1 } & Penicillium chrysogenum & 7 & Penicillium chrysogenum & 64.57 \\
\hline & Undetermined sp. & 1 & Emericella undulata & 13.98 \\
\hline & Nonsporulating sp. & 1 & Emericella olivicola & 10.46 \\
\hline \multirow[t]{4}{*}{ PG5-2 } & Alternaria alternata & 4 & Alternaria alternata & 31.33 \\
\hline & Aspergillus versicolor & 4 & Aspergillus subversicolor & 32.55 \\
\hline & & & Aspergillus puulaaeunsis & 36.12 \\
\hline & Nonsporulating sp. & 2 & & \\
\hline \multirow[t]{2}{*}{ PG5-3 } & Aspergillus versicolor & 6 & Aspergillus subversicolor & 39.24 \\
\hline & Penicillium chrysogenum & 8 & Penicillium chrysogenum & 60.76 \\
\hline
\end{tabular}

* PG = pulse group, i.e., the part of the air-conditioning system containing the machinery to pulse the air and the water-reservoir needed to maintain the required hygrometry level; the first number refers to the number of the PG (5 in total); the number after the '-' refers to the office connected to the PG, at different distances (see Materials and Methods). ${ }^{1} \mathrm{CFU} / \mathrm{mL}$ defined as the number of colonies observed on plate after five days and 21 days of incubation at $37^{\circ} \mathrm{C} .{ }^{2}$ The massive parallel sequencing data were analyzed with the CLC Genomic Workbench software (Qiagen Benelux, B.V., KJ Venlo, the Netherlands) and the Microbial Genomics Module. The database used as reference was the UNITE database ${ }^{36}$.

\subsection{RT-PCR Detection}

The RT-PCR screening was performed on DNA extracted from each air and water sample with the primers specific for the detection of E. jeanselmei only [31].

In each run, the PC was amplified with an average quantification cycle $(\mathrm{Cq})$ value of $19.48 \pm 1.08$ and a melting temperature $(\mathrm{Tm})$ average at $79.58 \pm 0.27^{\circ} \mathrm{C}$, corresponding to the expected values previously reported by Libert and colleagues [31]. Four of the five water samples gave a positive signal for E. jeanselmei with a Tm average at $79.63 \pm 0.30^{\circ} \mathrm{C}$, as seen in Table 1 . No signal was observed for the water sample collected from PG1.

Of the 15 indoor air samples tested, four of them gave a positive signal with the expected Tm, i.e., offices 1, 2, and 3 from PG4 and office 1 from PG5, as seen in Table 1. All the NTC were negative. Although the specificity of the E. jeanselmei RT-PCR primers has previously been demonstrated [31], the identity of each amplicon (i.e., amplicons from air and water samples) was confirmed as E. jeanselmei with a BLASTn analysis of the obtained Sanger sequences. The identify scores obtained with BLASTn for the eight positive samples ranged between 97\% for air sample PG4-2 and 100\% for the water samples from PGs 2 and 4.

\subsection{Massive Parallel Sequencing}

In order to confirm the results observed in RT-PCR and to obtain a broader view on the uncultivable and/or out-competed fraction in the air and water samples, a massive sequencing analysis was performed on all the DNA samples.

As shown in Table 4, the massive parallel analysis performed on all samples (water and air) generated between 6352 (sample PG1-3) and 29,666 reads (sample PG1-1). Among these reads, between 6797 and 11,602 reads from water samples were clustered into OTUs present in the UNITE database, corresponding to $60.97 \%$ (sample PG3) and $99.95 \%$ (PG5) of the total reads. 
Table 4. Detection of E. jeanselmei in indoor air and water in air-conditioning systems: sequencing data and clustering results.

\begin{tabular}{|c|c|c|c|c|c|c|c|c|}
\hline & $\begin{array}{l}\text { Pulse } \\
\text { Group }\end{array}$ & Office $^{1}$ & $\begin{array}{c}\text { Sample } \\
\text { Type }\end{array}$ & $\begin{array}{c}\text { Total } \\
\text { Number of } \\
\text { Reads }^{2}\end{array}$ & $\begin{array}{c}\text { Total of } \\
\text { Reads } \\
\text { Clustered }^{3}\end{array}$ & $\begin{array}{c}\% \\
\text { Reads } \\
\text { Clustered }^{4}\end{array}$ & $\begin{array}{l}\text { Abundance of } \\
\text { Reads into } \\
\text { E. jeanselmei } \\
\text { OTU }^{5}\end{array}$ & $\begin{array}{c}\% \text { Reads into } \\
\text { E. jeanselmei } \\
\text { OTU }(\%)^{6}\end{array}$ \\
\hline PG $1^{7}$ & 1 & & Water $^{8}$ & 13,124 & 11,602 & 88.40 & 0 & 0 \\
\hline PG1-1 & 1 & 1 & Air & 29,666 & 23,545 & 79.37 & 0 & 0 \\
\hline PG1-2 & 1 & 2 & Air & 19,753 & 19,603 & 99.24 & 0 & 0 \\
\hline PG1-3 & 1 & 3 & Air & 6352 & 6305 & 99.26 & 0 & 0 \\
\hline PG2 & 2 & & Water ${ }^{8}$ & 9755 & 9741 & 99.86 & 2727 & 28.00 \\
\hline PG2-1 & 2 & 1 & Air & 28,487 & 20,928 & 73.47 & 0 & 0 \\
\hline PG2-2 & 2 & 2 & Air & 15,193 & 15,093 & 99.34 & 0 & 0 \\
\hline PG2-3 & 2 & 3 & Air & 28,338 & 25,260 & 89.14 & 0 & 0 \\
\hline PG3 & 3 & & Water ${ }^{8}$ & 11,148 & 6797 & 60.97 & 2278 & 33.51 \\
\hline PG3-1 & 3 & 1 & Air & 9154 & 9003 & 98.35 & 0 & 0 \\
\hline PG3-2 & 3 & 2 & Air & 9474 & 9434 & 99.58 & 0 & 0 \\
\hline PG3-3 & 3 & 3 & Air & 9474 & 9165 & 96.74 & 0 & 0 \\
\hline PG4 & 4 & & Water ${ }^{8}$ & 10,675 & 10,616 & 99.45 & 5700 & 53.69 \\
\hline PG4-1 & 4 & 1 & Air & 8402 & 8360 & 99.50 & 5078 & 60.74 \\
\hline PG4-2 & 4 & 2 & Air & 16,838 & 16,837 & 99.99 & 7011 & 41.64 \\
\hline PG4-3 & 4 & 3 & Air & 20,286 & 20,059 & 98.88 & 7888 & 39.32 \\
\hline PG5 & 4 & & Water ${ }^{8}$ & 9302 & 8458 & 90.93 & 4453 & 52.65 \\
\hline PG5-1 & 5 & 1 & Air & 12,288 & 11,355 & 92.41 & 1248 & 10.99 \\
\hline PG5-2 & 5 & 2 & Air & 16,337 & 7016 & 42.95 & 0 & 0 \\
\hline PG5-3 & 5 & 3 & Air & 9328 & 9320 & 99.91 & 0 & 0 \\
\hline
\end{tabular}

The massive parallel sequencing data were analyzed with the CLC Genomic Workbench software (Qiagen Benelux, B.V., KJ Venlo, the Netherlands) and the Microbial Genomics Module. The database used as reference was the UNITE database ${ }^{36} .{ }^{1}$ Three offices per pulse group (PG) were sampled according to their distance to the PG. Office 1 corresponds to the closest located office to the PG, office 3 the most distantly located office to the PG, and office 2 correspond to the office located between the two others. ${ }^{2}$ The total number of reads observed for each sample. ${ }^{3}$ Number of total reads grouped in the 10 OTUs detected, i.e., A. alternata, Aspergillus fumigatus, Aspergillus monodii, Aspergillus puulaaeunsis, Aspergillus rugulosus, Aspergillus subversicolor, Emericella olivicola, Emericella undulata, P. chrysogenum, and E. jeanselmei. ${ }^{4}$ Percentage obtained as the ratio between the number of reads grouped into the 10 OTUs and the total number of reads observed for each sample. ${ }^{5}$ Number of reads corresponding to OTU identified as E. jeanselmei. ${ }^{6}$ Percentage obtained as the ratio between the number of reads corresponding to the E. jeanselmei OTU and the total number of clustered reads. ${ }^{7}$ PG1 maintained before the sampling. ${ }^{8}$ One sample per pulse group was analyzed.

For PG 2, 3, 4, and 5 (water samples), between 2727 and 5700 reads were grouped into the OTU and identified as E. jeanselmei strain AY156963, as seen in Table 4 and Figure 1, the only representative sequence for E. jeanselmei included in the UNITE database. The percentage of reads identified as E. jeanselmei was between 28\% (sample PG2) and 53.69\% (sample PG4) of the total number of clustered reads. No reads from PG1 were clustered into the E. jeanselmei OTU, as seen in Table 4.

The number of reads from air samples clustering into OTUs was between 6305 (sample PG1-3) and 25,260 (sample PG2-3). The percentage of reads clustered into OTUs was recorded between $42.95 \%$ (sample PG5-2) and 99.99\% (PG4-2). In four out of the 15 air samples, E. jeanselmei reads were found. Indeed, among the reads clustered into OTUs, between 1248 (sample PG5-1) and 7888 (sample PG4-3) reads were clustered into the E. jeanselmei AY156963 OTU, as seen in Table 4 and Figure 2. Expressed in \%, between $10.99 \%$ (sample PG5-1) and $60.74 \%$ (sample PG4-1) of reads grouped into OTUs were identified as E. jeanselmei OTU. These samples were collected in four offices connected to two PGs i.e., offices 1, 2, and 3 of PG4 and office 1 of PG5, as seen in Table 4. For the samples of PG1, 2, and 3, no reads were correctly clustered into the E. jeanselmei OTU, as seen in Table 4.

In addition to E. jeanselmei, as shown in Tables 2 and 3, additional OTUs were detected besides E. jeanselmei, i.e., three in water samples, A. fumigatus, A. puulaaeunsis and P. chrysogenum, as seen in Figure 1, and nine in air samples, i.e., A. alternata, Aspergillus monodii, A. puulaaeunsis, Aspergillus rugulosus, Aspergillus subversicolor, Aspergillus sp., Emericella olivicola, Emericella undulata, and P. chrysogenum, as seen in Figure 2. 


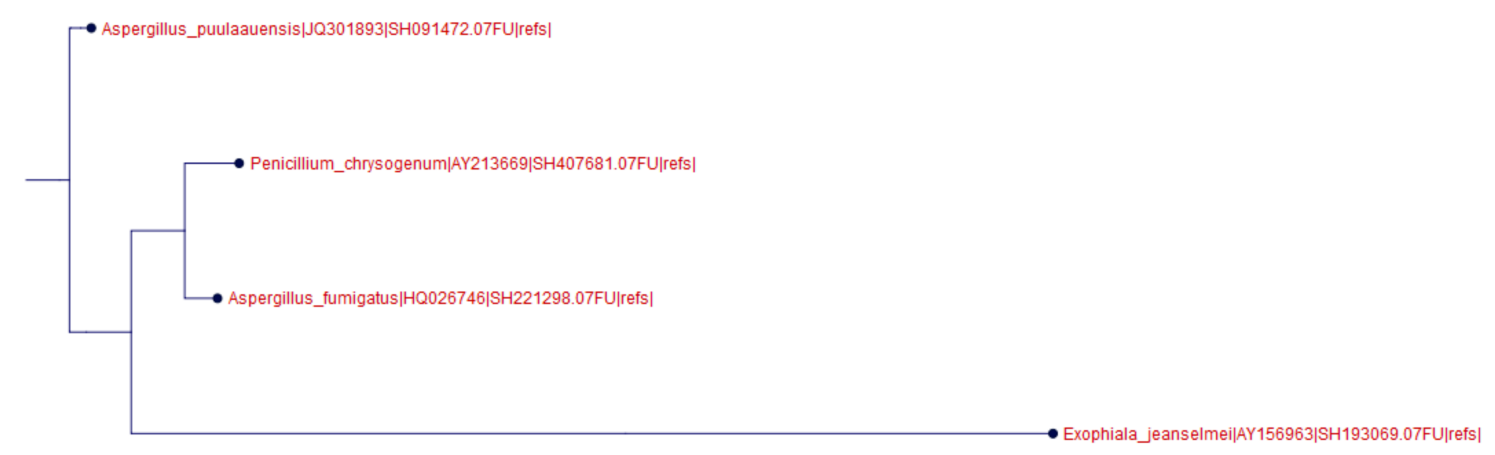

0.130

Figure 1. Neighbor Joining tree obtained for the water samples. The Neighbor Joining tree was constructed according to a Jukes Cantor model for the reads obtained for all water samples grouped together.

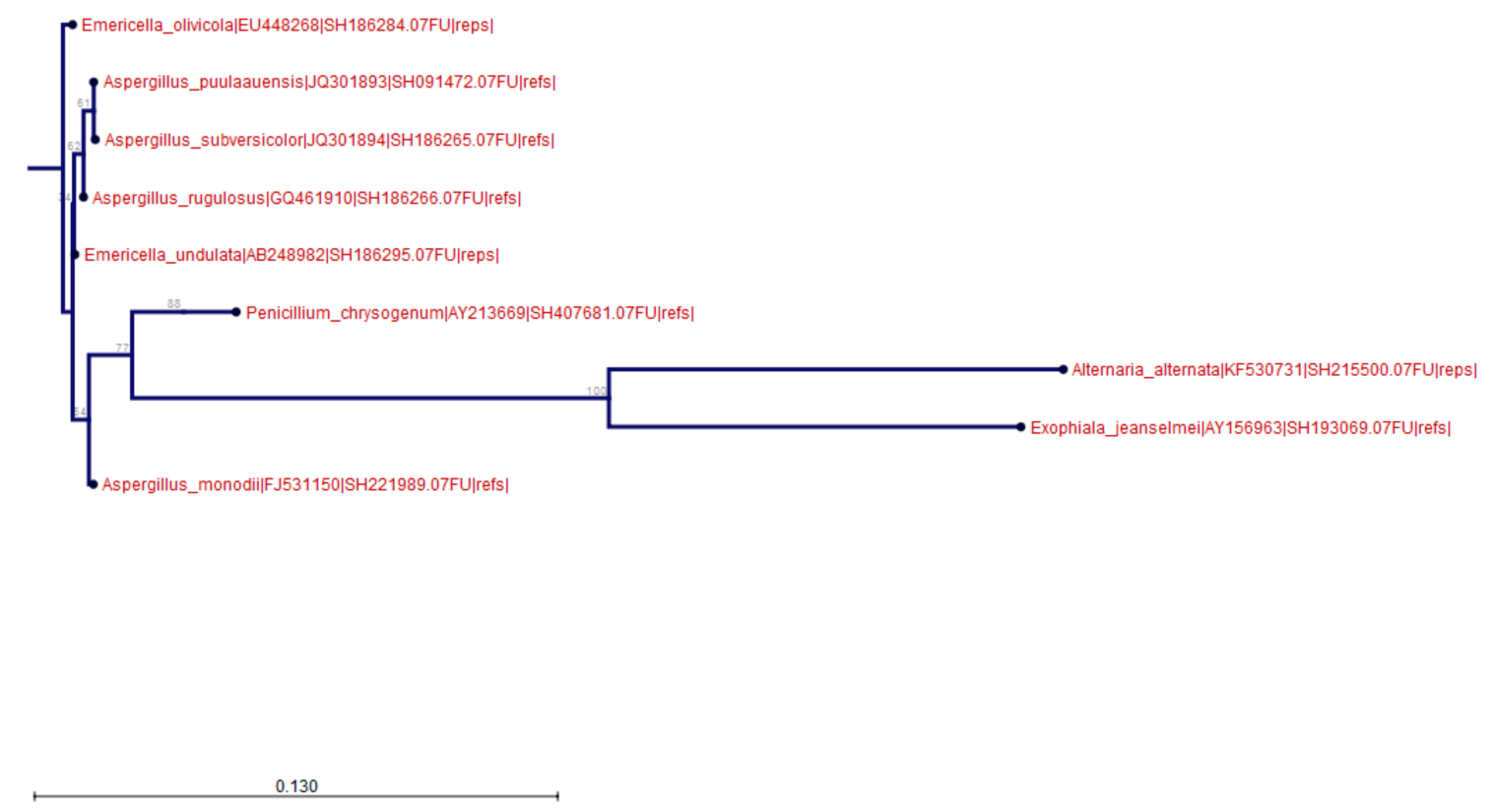

Figure 2. Neighbor Joining tree obtained for the air samples. The Neighbor Joining tree was constructed according to a Jukes Cantor model for the reads obtained for all air samples grouped together.

\section{Discussion}

E. jeanselmei is commonly found in moist environments, especially in water reservoirs of air-conditioning systems [37-39], but it has not yet been detected in indoor air of offices in contact with these air-conditioning systems. This could be explained by the classical use of culture-based protocols for the monitoring and investigation of fungal indoor air contamination. In this study, it was investigated whether the presence of E. jeanselmei in indoor air of these offices could be demonstrated using molecular methods, thereby avoiding the bias created by competition between species, incubation conditions, and the presence of uncultivable taxa when using culture-based methods.

Herein, a sampling was organized in different offices in contact with an air-conditioning system for which contamination by E. jeanselmei was previously detected. First, water samples from the 
water-reservoir from five PGs were collected in order to verify the presence of E. jeanselmei inside the system, i.e., in the water samples. These samples were analyzed using the classical protocol based on culture and microscopic identification [32], and molecular methods including RT-PCR [31] and massive parallel sequencing analysis. All samplings were performed between February and March, i.e., at the yearly restart of the air-conditioning systems, in the framework of the air-conditioning water monitoring. This monitoring is carried out yearly before the water decontamination (part of the maintenance of the system), except for PG1, which had been maintained (including decontamination and draining) already before the time of sampling. The classical analysis of the water samples showed that all of the investigated water systems were contaminated with E. jeanselmei, except the one from PG1, as seen in Table 1. The maintenance performed on PG1 explained the absence of E. jeanselmei. The results of the RT-PCR as well as of the massive parallel sequencing confirmed these positive detections of E. jeanselmei in water samples, as seen in Tables 1 and 4, of PG2, 3, 4, and 5. Consistently, neither a signal nor a read was observed for the PG1 sample with RT-PCR or massive parallel sequencing, respectively.

In parallel, indoor air samples were collected in 15 offices in contact with the air-conditioning systems. For each system, three offices were selected based on their distance to the pulse group, i.e., one close to the pulse group, one far from the pulse group and one in the middle of the two others. This was done in order to define whether a distance effect could impact the detection of E. jeanselmei in air. As performed for the water samples, each air sample was analyzed with classical and molecular approaches. No E. jeanselmei colonies were observed for the air samples incubated on plates, even after 21 days of incubation. However, positive signals for E. jeanselmei were observed by RT-PCR in air samples from the offices 1, 2, and 3 linked to PG4, and in office 1 of PG5. These positive detections were obtained for the air-conditioning systems where the highest water contamination was observed on plate (100 CFU/mL for PG4 and 15 CFU for PG5, compared to 10 for PG2 and PG3). In PG5, E. jeanselmei was observed only in the office the closest located to the PG, not in office 2 and 3 of PG5. Moreover, according to the RT-PCR results, a gradient of distance from the closest to the most distantly located offices was observed for the PG4 with Cq values ranging between $23.79 \pm 0.92$ for PG4-1 and $34.06 \pm 1.12$ for PG4-3. The distance between the source of the contamination and the sampling site and the contamination load could probably affect the spreading of biological material originating from E. jeanselmei as was observed for other species from indoor and outdoor environment [40]. It would be interesting to study the size and the form of the airborne biological compounds of E. jeanselmei involved to determine their aerosolizing distance in indoor air to verify this hypothesis.

The results of the massive parallel sequencing confirmed the ones obtained by RT-PCR. The obtained reads were clustered onto the UNITE database as reference, which contained 6825 reference ITS sequences at the date of research. Reads were clustered onto the E. jeanselmei OTU for samples PG4-1, PG4-2, PG4-3, and PG5-1. For these samples, positive RT-PCR signals were obtained. The percent of reads clustering into E. jeanselmei decreased with increasing distance between the sampling site and the PG location. This is a similar trend as was observed with the Cq values in the RT-PCR analysis. This points towards a semiquantitative nature of the massive parallel sequencing analysis.

Massive parallel sequencing was performed to obtain a broader view on the uncultivable species present in the water and air samples. Indeed, besides E. jeanselmei in indoor air and water samples, A. alternata, A. fumigatus, A. puulaaeunsis, A. versicolor, P. chrysogenum, as well as Aspergillus sp., an undetermined sp. and a nonsporulating sp., were detected on plate with the classical culture-based protocol. These taxa are commonly observed in indoor environment [1,33]. In the water samples, the sequencing and classical approaches yielded the same species. However, besides E. jeanselmei, five additional OTUs were retrieved from air with the massive parallel sequencing which were not detected by the classical methods in the air samples, i.e., A. monodii, A. puulaaeunsis, A. rugulosus, E. olivicola, and E. undulata. This means that of the species retrieved in the OTU analysis from air samples, only two were confirmed by the culturing approach, i.e., A. alternata and P. chrysogenum. A. versicolor was found in the air samples with the classical methods, but not with the massive parallel sequencing. 
However, two species from the same phylogenetic species, i.e., Versicolores were observed in the OTUs, i.e., A. puulaaeunsis and A. subversicolor. It should be noted that poor read quality could affect the correct determination of closely related species, e.g., for the species from the Versicolores complex and especially $A$. versicolor and $A$. puulaauensis, for which the ITS region differs only in a few nucleotides.

During the clustering, between $0.01 \%$ and $57.05 \%$ of reads were not included in the OTUs. This can be linked either to reads that did not comply with the quality criteria or to reads that correspond to species that are not covered in the UNITE database or chimeric reads. This indicates that the massive parallel sequencing approach would benefit from improved and extended ITS databases in the future. The lower number of clustered reads obtained for some samples, especially for samples PG3 and PG5-2, could have an impact on the results obtained during the diversity analysis. However, based on the RT-PCR results, these potential biases have not affected the detection of E. jeanselmei.

The literature available on E. jeanselmei detected in air is very scarce. In 2017, Kirtsideli and coworkers [20] detected this fungus for the first and only time in air, both outdoors (coastal areas, landscape, streets) and indoors (public building, empty houses). They showed that there was a higher concentration outdoors than indoors, when analyzing sampling data of 2012-2015 for the arctic settlement Tiksi, which suggests that the fungus could have a major outdoor source. During our monitoring campaign, unfortunately, no outdoor samples were taken. However, it has to be questioned whether the outdoor sampling on one day would be representative to explain a possible outdoor source of E. jeanselmei contamination, as was described for the 2012-2015 period in the arctic settlement [20]. Moreover, to the best of our knowledge, there are currently no reports available describing the detection using classical methods of E. jeanselmei in outdoor air samples in Belgium. Additionally, given the fact that E. jeanselmei was only found in four of the 15 sampled offices, with a clear correlation with a high PG contamination, we tend to believe that the source of air contamination in the sampled offices is the PG of the air-conditioning system. In 2010, Huang and his team reported a case of hypersensitivity pneumonitis caused by E. jeanselmei in a person who was a regular visitor of a specific sauna [41]. According to their conclusion, the way of contamination was probably by the sauna steam [41], due to the presence of the black yeast in the water of the sauna visited by the patient. However, as this black yeast can be present in dry indoor air from buildings, this could also be a possible way of infection.

The detection of E. jeanselmei with molecular tools, but not with the classical method, confirms the hypothesis that E. jeanselmei could be present in the suppressed or uncultivable fraction of the indoor airborne fungal community. This indicates that airborne infection of E. jeanselmei is possible. As shown in this study, RT-PCR and massive parallel sequencing analysis can improve the monitoring of indoor air, especially in the field of fungal contamination. Indeed, the use of universal primers amplifying the ITS region of a large panel of fungal species increases the number of species that can be simultaneously detected during a monitoring. This is limited to one or a few a priori selected species with RT-PCR [42]. Another advantage of massive parallel sequencing is that the detection and identification are performed simultaneously, while the confirmation of RT-PCR results requires sequencing of the amplicon if the tool is not fully specific. However, massive parallel sequencing is more time-consuming and requires bioinformatic expertise for the data interpretation, which is not the case for RT-PCR. In addition to the use of molecular tools, the detection of E. jeanselmei was probably also facilitated by the use of this particular air collector, which is different from the one generally used for culture-dependent monitoring. The Coriolis ${ }^{\circledR} \mu$ allows collecting a high volume of air $\left(1.5 \mathrm{~m}^{3}\right)$ directly into collection liquid, which can be incubated on a plate or analyzed with molecular tools without a culturing step, as was done in this study.

\section{Conclusions}

The results obtained in this study, taking E. jeanselmei as a case study, demonstrate that molecular methods, such as RT-PCR or massive parallel sequencing, combined with an appropriate air sampler, can increase the data retrieved on the fungal community diversity in indoor air, compared to the classical culture-based methods. More investigations on indoor air should be performed in order 
to improve the knowledge on indoor airborne fungi contamination, particularly on the suppressed and/or uncultivable fraction. Eventually, an improved insight on this diversity will contribute to the understanding of public health problems such as respiratory diseases (asthma, rhinitis, or other chronicle respiratory infections) and sick buildings syndrome observed in many indoor environments, and to taking the appropriate preventive measures.

Author Contributions: Conceptualization, C.C., A.P., N.H.R. and S.C.J.D.K.; Data curation, X.L.; Formal analysis, X.L., C.C. and S.C.J.D.K.; Funding acquisition, S.C.J.D.K.; Investigation, X.L. and C.C.; Methodology, C.C., A.P., N.H.R. and S.C.J.D.K.; Project administration, S.C.J.D.K.; Resources, A.P., F.B., N.H.R. and S.C.J.D.K.; Supervision, N.H.R. and S.C.J.D.K.; Validation, X.L., C.C. and S.C.J.D.K.; Visualization, X.L.; Writing—original draft, X.L. and S.C.J.D.K.; Writing—review \& editing, X.L., C.C., A.P., F.B., N.H.R. and S.C.J.D.K.

Funding: This research was funded by RP/PJ WIV-ISP (mycoMOLAIR).

Acknowledgments: We would like to thank the technicians from the Environmental and Health service from Sciensano for the classical analysis. We greatly acknowledge Amaury de Maere from Vinçotte (Belgium) and Dirk Van Geel from Transversal activities in Applied Genomics (Sciensano) for their help during the sampling.

Conflicts of Interest: The authors declare no conflict of interest. The funders had no role in the design of the study; in the collection, analyses, or interpretation of data; in the writing of the manuscript, or in the decision to publish the results.

\section{References}

1. Bellanger, A.P.; Reboux, G.; Roussel, S.; Grenouillet, F.; Didier-Scherer, E.; Dalphin, J.C.; Millon, L. Indoor fungal contamination of moisture-damaged and allergic patient housing analysed using real-time PCR. Lett. Appl. Microbiol. 2009, 49, 260-266. [CrossRef] [PubMed]

2. Mendell, M.J.; Mirer, A.G.; Cheung, K.; Tong, M.; Douwes, J. Respiratory and allergic health effects of dampness, mold, and dampness-related agents: A review of the epidemiologic evidence. Env. Health Perspect. 2011, 119, 748-756. [CrossRef] [PubMed]

3. Packeu, A.; Chasseur, C.; Bladt, S.; Detandt, M. The role of indoor pollution in the development and maintenance of chronic airway inflammation in children. B-ENT 2012, 8, 73-79. [PubMed]

4. Meheust, D.; Le Cann, P.; Reboux, G.; Millon, L.; Gangneux, J.P. Indoor fungal contamination: Health risks and measurement methods in hospitals, homes and workplaces. Crit. Rev. Microbiol. 2014, 40, 248-260. [CrossRef]

5. Portnoy, J.M.; Jara, D. Molds allergy revisited. Ann. Allergy Asthma Immunol. 2015, 114, 83-89. [CrossRef]

6. Dannemiller, K.C.; Gent, J.F.; Leaderer, B.P.; Peccia, J. Indoor microbial communities: Influence on asthma severity in atopic and nonatopic asthma. J. Allergy Clin. Immunol. 2016, 138, 76-83. [CrossRef]

7. European Environment Agency. Indoor Air Quality. Available online: http://www.eea.europa.eu/signals/ signals-2013/articles/indoor-air-quality (accessed on 15 October 2019).

8. United States Environmental Protection Agency. An introduction to indoor air quality. Available online: https://www.epa.gov/indoor-air-quality-iaq (accessed on 15 October 2019).

9. WHO Regional Office for Europe. WHO Guidelines for Indoor Air Quality: Dampness and Mould; World Health Organization: Copenhagen, Denmark, 2009.

10. Shelton, B.G.; Kirkland, K.H.; Flanders, W.D.; Morris, G.K. Profiles of airborne fungi in buildings and outdoor environments in the United States. Appl. Env. Microbiol. 2002, 68, 1743-1753. [CrossRef]

11. Horner, W.E.; Worthan, A.G.; Morey, P.R. Air- and dustborn mycoflora in houses free of water damage and fungal growth. Appl. Env. Microbiol. 2004, 70, 6394-6400. [CrossRef]

12. Kelkar, U.; Bal, A.; Kulkarni, S. Fungal contamination of air conditionning units in operating theatres in India. JHI 2005, 60, 81-84. [CrossRef]

13. Ponsoni, K.; Gonçalves Raddi, M.S. Indoor air quality related to occupancy at an air-conditioned public health. Braz. Arch Biol. Technol. 2010, 53, 99-103. [CrossRef]

14. Adams, R.I.; Miletto, M.; Taylor, J.W.; Bruns, T.D. Dispersal in microbes: Fungi in indoor air are dominated by outdoor air and show dispersal limitation at short distances. ISME J. 2013, 7, 1262-1273. [CrossRef] [PubMed] 
15. Nucci, M.; Akiti, T.; Barreiros, G.; Silveira, F.; Revankar, S.; Sutton, D.; Patterson, T. Nosocomial fungemia due to Exophiala jeanselmei var. jeanselmei and a Rhinocladiella species: Newly described causes of bloodstream infection. J. Clin. Microbiol. 2001, 39, 514-518. [CrossRef] [PubMed]

16. Wang, L.; Yokoyama, K.; Miyaji, M.; Nishimura, K. Identification; classification; and phylogeny of the pathogenic species Exophiala jeanselmei and related species by mitochondrial cytochrome $\mathrm{b}$ gene analysis. J. Clin. Microbiol. 2001, 39, 4462-4467. [CrossRef] [PubMed]

17. Nucci, M.; Akiti, T.; Barreiros, G.; Silveira, F.; Revankar, S.; Wickes, B.; Sutton, D.; Patterson, T. Nosocomial outbreak of Exophiala jeanselmei fungemia associated with contamination of hospital water. Clin. Infect Dis. 2002, 34, 1475-1480. [CrossRef] [PubMed]

18. Zeng, J.; Sutton, D.; Fothergill, A.; Rinaldi, M.; Harrak, M.; de Hoog, G. Spectrum of clinically relevant Exophiala species in the United States. J. Clin. Microbiol. 2007, 45, 3713-3720. [CrossRef]

19. Sterflinger, K. Black yeasts and meristemactic fungi: Ecology; diversity and identification. In Biodiversity and Ecophysiology of Yeasts; Péter, G., Rosa, C., Eds.; Springer: Berlin/Heidelberg, Germany, 2009; Volume 20, pp. 501-514.

20. Kirtsideli, I.Y.; Vlasov, D.Y.; Abakumov, E.V.; Barantsevich, E.P.; Novozhilov, Y.K.; Krylenkov, V.A.; Sokolov, V.T. Airborne fungi in arctic settlement Tiksi (Russian Arctic, coast of the Laptev Sea). Czech Polar Rep. 2017, 7, 300-310. [CrossRef]

21. Nishimura, K.; Miyaji, M.; Taguchi, H.; Tanaka, R. Fungi in bathwater and sludge of bathroom drainpipes. Frequent isolation of Exophiala species. Mycopathologia 1987, 97, 17-23. [CrossRef]

22. Badali, H.; Chander, J.; Bayat, M.; Seyedmousavi, S.; Sidhu, S.; Rani, H.; Attri, A.; Handa, U.; Meis, J.; de Hoog, G. Multiple subcutaneous cysts due to Exophiala spinifera in an immunocompetent patient. Med. Mycol. 2012, 50, 207-213. [CrossRef]

23. Pitkaranta, M.; Meklin, T.; Hyvarinen, A.; Nevalainen, A.; Paulin, L.; Auvinen, P.; Lignell, U.; Rintala, H. Molecular profiling of fungal communities in moisture damaged buildings before and after remediation-A comparison of culture-dependent and culture-independent methods. BMC Microbiol. 2011, 11, 235. [CrossRef]

24. Vesper, S.J. Traditional mould analysis compared to a DNA-based method of mould analysis. Crit. Rev. Microbiol. 2011, 37, 15-24. [CrossRef]

25. Anaissie, E.J.; Stratton, S.L.; Dignagi, M.C.; Lee, C.K.; Summerbell, R.C.; Rex, J.H.; Monson, T.P.; Walsh, T.J. Pathogenic molds (including Aspergillus species) in hospital water distribution systems: A 3-year prospective study and clinical implications for patients with hematologic malignancies. Blood 2003, 101, 2542-2546. [CrossRef] [PubMed]

26. Haugland, R.A.; Varma, M.; Wymer, L.J.; Vesper, S.J. Quantitative PCR analysis of selected Aspergillus; Penicillium and Paecilomyces species. Syst. Appl. Microbiol. 2004, 27, 198-210. [CrossRef] [PubMed]

27. Timothy, D.; Betancourt, D.; Menetrez, M. A rapid DNA extraction method for PCR identification of fungal indoor air contaminants. J. Microbiol. Methods 2004, 56, 431-434.

28. Michealsen, A.; Pinzari, F.; Ripka, K.; Lubitz, W.; Piñar, G. Application of molecular techniques for identification of fungal communities colonising paper materials. Int. Biodeter. Biodegrad. 2006, 58, 133-141. [CrossRef]

29. Reboux, G.; Bellanger, A.P.; Roussel, S.; Grenouillet, F.; Sornin, S.; Piarroux, R.; Dalphin, J.C.; Millon, L. Indoor mold concentration in Eastern France. Indoor Air. 2009, 19, 446-453. [CrossRef] [PubMed]

30. Hospodsky, D.; Yamamoto, N.; Peccia, J. Accuracy; precision; and method detection limits of quantitative PCR for airborne bacteria and fungi. Appl. Env. Microbiol. 2010, 76, 7004-7012. [CrossRef]

31. Libert, X.; Chasseur, C.; Packeu, A.; Bureau, F.; Roosens, N.H.; De Keersmaecker, S.C.J. A molecular approach for the rapid; selective and sensitive detection of Exophiala jeanselmei in environmental samples: Development and performance assessment of a real-time PCR assay. Appl. Microbiol. Biotechnol. 2016, 100, 1377-1392. [CrossRef]

32. Nolard, N.; Chasseur, C.; Marlier, M.; Lognay, G. Validation des Méthodes Microbiologiques et Chimiques de Contrôle des Lieux de Travail. 2004. Available online: http://www.belspo.be/belspo/organisation/publ/ pub_ostc/PS/rPS19r_fr.pdf (accessed on 15 October 2019).

33. Libert, X.; Chasseur, C.; Bladt, S.; Packeu, A.; Bureau, F.; Roosens, N.; De Keersmaecker, S.C.J. Development and validation of a qualitative SYBR ${ }^{\circledR}$ green real-time PCR assay for the detection of Aspergillus versicolor from indoor air. Appl. Microbiol. Biotechnol. 2015, 99, 7267-7282. [CrossRef] 
34. White, T.; Bruns, T.; Lee, S.; Taylor, J. Amplification and direct sequencing of fungal ribosomal RNA genes for phylogenetics. In PCR Protocols: A Guide to Methods and Applications; Innis, M., Gelfand, D., Shinsky, J., White, T., Eds.; Academic Press: Amsterdam, The Netherlands, 1990; pp. 315-322.

35. Andrews, S. FastQC: A Quality Control Tool for High Throughput Sequence Data. [Babraham Bioinformatics Website]. October 2010. Available online: http://www.bioinformatics.babraham.ac.uk/projects/fastqc (accessed on 10 September 2019).

36. Kõljalg, U.; Larsson, K.H.; Abarenkov, K.; Nilsson, R.H.; Alexander, I.J.; Eberhardt, U.; Erland, S.; Høiland, K.; Kjøller, R.; Larsson, E.; et al. UNITE: A database providing web-based methods for the molecular identification of ectomycorrhizal fungi. New Phytol. 2005, 166, 1063-1068.

37. Beguin, H.; Nolard, N. Mould biodiversity in homes. I. Air and surface analysis of 130 dwellings. Aerobiologia 1994, 10, 157-166. [CrossRef]

38. Heinemann, S.; Beguin, H.; Nolard, N. Biocontamination in air-conditioning. In Health Implication of Fungi in Indoor Environment; Samson, R.A., Flannigan, B., Flannigan, M.E., Verhoeff, A.P., Adan, O.C.G., Hoekstra, E.S., Eds.; Elsevier: Amsterdam, The Netherlands, 1994; pp. 179-186.

39. Parat, S.; Fricker-Hidalgo, H.; Perdrix, A.; Bemer, D.; Pelissier, N.; Grillot, R. Airborne fungal contamination in air-conditionning systems: Effect of filtering and humidifying devices. AIHA J. 1996, 57, 996-1001. [CrossRef]

40. Afanou, K.A.; Straumfors, A.; Skogstad, A.; Nayak, A.P.; Skaar, I.; Hejeljord, L.; Transmo, A.; Eduard, W.; Green, B.J. Indirect immunodetection of fungal fragments by field emission scanning electron microscopy. Appl. Env. Microbiol. 2015, 1, 5794-5803. [CrossRef] [PubMed]

41. Huang, W.C.; Lu, Y.H.; Lin, Z.G.; Su, W.L. Sauna lung: Hypersensitivity pneumonitis due to Exophiala jeanselmei. Respiro Case Rep. 2010, 15, 573-579. [CrossRef] [PubMed]

42. Gurvich, O.L.; Skoblov, M. Real-Time PCR and Multiplex Approaches. Methods Mol. Biol. 2011, 2, 1-13. [CrossRef]

(C) 2019 by the authors. Licensee MDPI, Basel, Switzerland. This article is an open access article distributed under the terms and conditions of the Creative Commons Attribution (CC BY) license (http://creativecommons.org/licenses/by/4.0/). 